\title{
KIF20A mRNA and Its Product MKIp2 Are Increased During Hepatocyte Proliferation and Hepatocarcinogenesis
}

\author{
Isabelle Gasnereau, ${ }^{\star \dagger \ddagger}$ Mathieu Boissan, ${ }^{* \neq \S}$ \\ Germain Margall-Ducos, ${ }^{\text {III }}$ Gabrielle Couchy, ${ }^{* \star+\dagger}$ \\ Dominique Wendum, ${ }^{\star \neq \ddagger}$ \\ Florence Bourgain-Guglielmetti, ${ }^{* \dagger}$ \\ Chantal Desdouets, ${ }^{\text {III }}$ Marie-Lise Lacombe, ${ }^{\star \S}$ \\ Jessica Zucman-Rossi, ${ }^{* * \dagger+}$ and \\ Joëlle Sobczak-Thépot ${ }^{\star \dagger}$

\begin{abstract}
From the Universite Pierre et Marie Curie (UPMC),* Paris; the Laboratoire de Biologie du Développement, ${ }^{\dagger}$ Centre National de la Recherche Scientifique (CNRS) UMR 7622, Paris; the Service de Biochimie et Hormonologie, ${ }^{\ddagger}$ Assistance Publique -Hôpitaux de Paris (AP-HP), Hôpital Tenon, Paris; INSERM UMR_S938, Centre de Recherches Saint-Antoine, ${ }^{\Omega}$ Paris; Institut Cochin, ${ }^{\text {"I }}$ Université Paris Descartes, CNRS (UMR 8104), Paris; INSERM U567", Paris; INSERM U674,** Paris; the Département d'Oncologie, ${ }^{\text {t† }}$ Université Paris Descartes, AP-HP, Hôpital Européen Georges Pompidou, Paris; and the Service d'Anatomo-Pathologie, ${ }^{\#}$ AP-HP, Hôpital Saint-Antoine, Paris, France
\end{abstract}

Mitotic kinesin-like protein 2 (MKlp2), a microtubule-associated motor, is required during mitosis exit for the final step of cytokinesis. It also contributes to retrograde vesicular trafficking from the Golgi apparatus to the endoplasmic reticulum in interphase. The KIF2OA gene encoding MKlp2 is controlled by the E2F-retinoblastoma proteinp16 pathway, and its widely expressed mRNA is found in fetal and proliferating adult tissues. The expression pattern and function of MKlp2 in the adult liver, however, have not been investigated. We report herein that MKlp2 transiently accumulates in vivo during mouse liver regeneration after partial hepatectomy and is strongly overexpressed in preneoplastic and neoplastic mouse liver. In vitro in mitogen-stimulated primary hepatocytes, MKlp2 accumulated in the nucleus during the $G 2$ phase of the cell cycle coincident with the mitotic kinase Aurora B. Human hepatoma cell lines exhibited high levels of MKlp2; however, it was undetectable in normal human hepatocytes. RNAi-mediated MKlp2 knockdown in hepatoma cells induced polyploidization consistent with its essential function in promoting cytokinesis and inhibited cell proliferation without inducing apoptosis. KIF20A mRNA was strongly accumulated in a large series of human hepatocellular carcinomas, with the highest expression observed in tumors with genomic instability. Accumulation of MKlp2 in normal proliferating, preneoplastic, and transformed hepatocytes suggests that MKlp2 contributes to both normal and pathologic hepatocyte proliferation and is linked to tumor aggressiveness in human hepatocellular carcinomas. (Am J Pathol 2012, 180:131-140; DOI: 10.1016/j.ajpath.2011.09.040)

Microtubule-associated motor proteins have important roles in numerous cellular processes including intracellular traffic and cell division. In Saccharomyces cerevisiae, five kinesins are essential for formation, orientation, and elongation of the mitotic spindle and for segregation of chromosomes, whereas in human cells, at least 12 kinesins participate in regulating mitosis and cytokinesis. ${ }^{1}$ Among these, the mitotic kinesin-like proteins MKIp1 and MKIp2, two closely related members of the kinesin- 6 family, are required for cytokinesis. ${ }^{2}$ MKIp1 interacts with several essential regulators of cytokinesis such as the complex of passenger proteins, the regulators of RhoA ECT2 and MgcRacGAP (CYK-4), and Polo-like kinase 1 (Plk-1), and moves them from kinetochores in metaphase to the central spindle in anaphase, where they remain until cytokinesis. MKIp2 cooperates with MKIp1 by recruiting and restricting the activities of Plk1 and Aurora B kinases and of CDC14A phosphatase to the central spin-

Supported by grants from University Paris 6 and CNRS (J.S.T.), INSERM and La Ligue Comité de Paris (RSO6/75-57 to C.D.), INSERM and Ligue Nationale Contre le Cancer CIT project (J.Z.R.). I.G. and G.M.D. were fellows of the Ministère de la Recherche et de la Technologie and Association pour la Recherche Contre le Cancer.

Accepted for publication September 13, 2011.

I.G. and M.B. contributed equally to this work.

Supplemental material for this manuscript can be found at $h t t p: / / a j p$. amjpathol.org or at doi: 10.1016/j.ajpath.2011.09.040.

Address reprint requests to Joëlle Sobczak-Thépot, Ph.D., UPMCUniversité Paris 6, Laboratoire de Biologie du Développement, CNRS UMR 7622, 9 Quai Saint-Bernard, case 24, 75005 Paris, France. E-mail: Joelle.Sobczak_Thepot@upmc.fr. 
dle during anaphase. ${ }^{3-5}$ MKIp2 is required for cell abscission, the final step in cytokinesis. ${ }^{1,5-7}$ During interphase, MKIp2 participates in retrograde vesicular traffic between the Golgi apparatus and the endoplasmic reticulum. $^{8}$ This interphasic activity is linked to the ability of MKIp2 to interact with GTP-bound forms of Rab6; hence it is also named rabkinesin-6. Levels and activity of MKIp2 could, therefore, participate in regulation of either or both intracellular transport and cytokinesis. Little is known about the regulation of MKIp2 expression. The mRNA is widely expressed in human fetal tissues and is particularly abundant in fetal liver and thymus. It is also expressed in the adult during lymphopoiesis and hematopoiesis and in several proliferating tissues such as thymus and testis. ${ }^{9}$ However, no expression of MKIp2 is detectable in the adult quiescent human liver.

Under physiologic conditions, the liver is quiescent. In the adult, hepatocytes are either mononucleate or binucleate and exhibit various levels of ploidy. However, hepatocytes retain the unique property to self-renew and to repair the liver ad integrum when stimulated to proliferate after liver injury. After partial hepatectomy or liver damage, hepatocytes enter the cell cycle and proliferate to restore liver mass and function. ${ }^{10}$ Precise control of hepatocyte proliferation is critical for suppression of tumorigenesis in the liver, and, in turn, liver tumorigenesis is favored after chronic liver damage, stimulating cycles of liver regeneration. Deregulation of cell cycle genes, disruption of the retinoblastoma protein (pRb) pathway, and loss of expression of the liver-enriched transcription factor $\mathrm{HNF} 4 \alpha$ are frequent features of liver tumors, which are often accompanied by nuclear enlargement and increased or aberrant ploidy. ${ }^{11-16}$ MKIp2 as a potential target of both the pRb pathway ${ }^{6}$ and the HNF4 $\alpha$ pathway ${ }^{17}$ involved in cytokinesis could be an attractive target for anticancer therapy.

In the present study, we demonstrate that MKIp2 is overexpressed during normal, preneoplastic, and neoplastic hepatocyte proliferation, which suggests that MKIp2 contributes to both normal and pathologic hepatocyte proliferation. Inhibition of MKIp2 expression by RNA interference induces polyploidization of human hepatoma cells and inhibits cell proliferation without inducing apoptosis. In addition, in a large series of human hepatocarcinomas, we observed a dramatic increase in KIF20A mRNA levels linked to genomic instability.

\section{Materials and Methods}

\section{Animal Models}

For partial hepatectomy, 3- to 4-month-old 129/Sv male mice were purchased from Charles River Laboratories France SAS (L'Arbresle, France). Mice were anesthetized via intraperitoneal injection of xylazine $(4.8 \mathrm{mg} / \mathrm{kg}$ body weight; Bayer Schering Pharma AG, Berlin, Germany) and ketamine (72 mg/kg body weight; Merial SAS, Lyon, France), and underwent resection of two-thirds of the liver. After surgery, animals were maintained under standard conditions. Transgenic ASV mice (C57BL/6J-DBA2) harboring the SV40 large $T$ antigen on the $Y$ chromosome under the control of the human antithrombin III regulatory se- quences have been described previously. ${ }^{18,19}$ In this model, all male animals develop hepatocellular carcinoma (HCC). Nontransgenic male mice were used as controls. Animal experiments were conducted in accordance with national ethical guidelines for the care and use of laboratory animals.

\section{Isolation of Primary Mouse Hepatocytes}

Primary hepatocytes were isolated from 4-week-old C57B6J mice via liver perfusion using a collagenase blend (Liberase; Roche Applied Science, Meylan, France) and then were placed in the mitogenic stimulation medium as previously described. ${ }^{20}$ Cells were processed for immunofluorescence or were scrapped and frozen for protein analysis.

\section{Ki-67 Immunolabeling on Mouse Liver Sections}

After partial hepatectomy, the percentage of hepatocyte proliferation was evaluated using Ki-67 immunolabeling of liver sections using a SuperSensitive Link-Label Immunohistochemistry Detection System (BioGenex Laboratories, Inc., San Ramon, CA) according to the manufacturer's protocol using anti-Ki-67 antibody (1:100; Novocastra Laboratories, Ltd., Newcastle-upon-Tyne, England). A microscope eyepiece with a net micrometer (Carl Zeiss AG, Jena, Germany) at high magnification $(\times 400)$ was used to count Ki-67-positive hepatocyte nuclei in 10 consecutive fields from each liver section $\left(0.96 \mathrm{~mm}^{2}\right)$. Six to eight animals were analyzed at each time point. Tumoral livers from ASV mice were treated the same way.

\section{Human Hepatoma Cell Lines and Primary Hepatocytes}

Human hepatoma cell lines (HuH6, HuH7, PLC/PRF/5, HepG2, and Hep3B) were maintained in minimal essential medium containing Earle's salts, $1 \%$ nonessential amino acids, $1 \mathrm{mmol} / \mathrm{L}$ sodium pyruvate, and $10 \%$ fetal calf serum. Human primary hepatocytes were prepared as reported elsewhere. ${ }^{21}$

\section{RNA Interference}

Double-stranded short hairpin RNA (shRNA) corresponding to the cDNA sequence of human KIF2OA (5'-AAGATCAGGGTTGTGTCCGTAT-3') was from 251 to 273 relative to the first nucleotide of the coding sequence. No cross-reactive sequence other than the gene of interest was observed after blast analysis against the human genome. DNA oligonucleotides were purchased from Eurogentec SA (Seraing, Belgium), annealed, and subcloned in the pSuper vector as described. ${ }^{22} \mathrm{~A}$ pSuper containing a scrambled sequence inefficient in cells served as control. Cells were electroporated and collected after 36 hours for Western blot analysis and flow cytometric determination of DNA content as previously described. ${ }^{23}$ To quantify the percentages of cells with $2 \mathrm{n}$, $4 n$, and $>4 n$ DNA contents, modeling of the cell cycle profiles was performed using Multicycle software (Phoe- 
nix Flow Systems, Inc., San Diego, CA) in the two-cycle mode. The data were interpreted as percentages of cells in a diploid or tetraploid cycle. Apoptosis was detected 36 hours after transfection with annexin V-FITC and propidium iodide as recommended by the manufacturer (Apoptosis Detection Kit; Sigma-Aldrich Corp., St. Louis, MO). For the colony-formation assay, HuH6 cells were plated at $10^{5}$ cells per $25-\mathrm{cm}^{2}$ flask at 24 hours after co-transfection with the pSuper vector expressing either MKIp2 or control shRNA and a neo expressing vector. Cells stably expressing shRNA were selected for 14 days in the presence of 0.5 $\mathrm{mg} / \mathrm{mL}$ G418. Cells and colonies were imaged and analyzed using Adobe Photoshop after fixing with 100\% methanol and staining with May-Grünwald-Giemsa.

\section{MKIp2 Expression in Human HCCs}

Tumoral and nontumoral adjacent liver tissue specimens were collected from five patients with HCC who underwent partial hepatectomy without previous antitumor treatment. Histologic analyses of nontumoral adjacent liver tissues demonstrated no fibrosis in three patients (patients 1, 3, and 4) and cirrhosis in two (patients 2 and 5). Chronic liver disease was related to hepatitis $C$ virus infection in two patients (patients 2 and 5). All liver tissue samples were stored at $-80^{\circ} \mathrm{C}$ until analysis. Portions of the same livers were used for protein extraction, Western blot analysis, and immunohistochemistry.

\section{Quantitative RT-PCR Analysis of KIF20A mRNA in Human HCCs}

All of the $125 \mathrm{HCCs}$ and 21 nontumor samples have been previously described by Boyault et $\mathrm{al}^{24}$ in accordance with French law and institutional ethical guidelines. In brief, tissues were immediately snap-frozen in liquid nitrogen and stored at $-80^{\circ} \mathrm{C}$ from resected or explanted HCCs from patients who underwent treatment in France from 1991 to 2001. The primary clinical and pathologic features are given in Table 1. Mutations in the TP53 gene and fractional allelic loss were analyzed as previously described. ${ }^{24}$ RNA extraction and quantitative RT-PCR were performed as previously described. ${ }^{24}$ In brief, $1 \mu \mathrm{g}$ total RNA was reverse transcribed using the High Capacity Archive Kit and random hexamers (Applied Biosystems, Inc., Foster City, CA), and $5 \mu \mathrm{L}$ cDNA corresponding to $10 \mathrm{ng}$ reverse-transcribed RNA were analyzed using TaqMan PCR analysis, in duplicate, using the $A B I$ PRISM 7900HT System (Applied Biosystems, Inc.). Previously developed sequence detection reagents specific for the human KIF20A gene (Applied Biosystems; ref Hs00194882_m1) were used as described ${ }^{25}$ using the $2^{-\Delta \Delta \mathrm{CT}}$ method. ${ }^{26}$ Statistical analyses were performed using PRISM software (version 4; GraphPad Software, Inc., San Jose, CA), and significance was determined using the nonparametric Mann-Whitney $U$ test. All values reported are given as mean \pm SEM. Difference was considered significant at $P<0.05$.
Table 1. Clinical and Pathologic Characteristics of 125 Human HCCs Analyzed for KIF20A mRNA*

\begin{tabular}{lc}
\hline Clinical or pathologic characteristic & $\begin{array}{c}\text { HCC tumors } \\
(n=125)\end{array}$ \\
\hline Age, mean (SD), years & $60(13)$ \\
Sex (M/F) & $4: 1$ \\
$\alpha$-Fetoprotein <100 & 64 \\
Cirrhosis or hepatic adenoma & 68 \\
HBV infection & 30 \\
HCV infection & 27 \\
Alcoholism & 34 \\
Hemochromatosis & 5 \\
Diameter, mean (SD), cm & $7.6(4.5)$ \\
Edmondson grade III or IV & 42 \\
Microscopic vascular emboli & 37 \\
TP53 mutations & 26 \\
Chromosome instability (FAL > 0.128) & 46 \\
\hline *Unless otherwise indicated, values are given as percent. \\
FAL, fractional allelic loss; HBV, hepatitis B virus; HCV, hepatitis C virus.
\end{tabular}

\section{Protein Extraction and Immunoblot Analysis}

Proteins were extracted in RIPA buffer [ $50 \mathrm{mmol} / \mathrm{L}$ Tris $\mathrm{HCl}(\mathrm{pH}$ 7.5), $150 \mathrm{mmol} / \mathrm{L} \mathrm{NaCl}, 1 \% \mathrm{NP}-40,0.1 \% \mathrm{SDS}, 0.5 \%$ deoxycholate, $10 \mathrm{mmol} / \mathrm{L} \beta$-glycerophosphate, $1 \mathrm{mmol} / \mathrm{L} \mathrm{NaF}, 0.1$ $\mathrm{mmo} / \mathrm{L} \mathrm{Na}_{3} \mathrm{VO}_{4}, 1 \mathrm{mmo} / \mathrm{L}$ dithiothreitol, and protease inhibitors], followed by sonication $\left(4^{\circ} \mathrm{C}, 9 \times 30\right.$ seconds, $\left.80 \%\right)$. After electrophoresis and transfer, nitrocellulose membranes (Protran BA83; Whatman GmbH, Dassel, Germany) were incubated with antibodies against MKIp2 (anti-rabkinesin-6, 1:1000, Echard et al ${ }^{8}$ ), Aurora B (1:250; AlM1, Cell Signaling Technology, Inc., Beverly, MA), Importin- $\beta 2$ (1:200, D45; Santa Cruz Biotechnology, Inc., Santa Cruz, CA), PCNA (1:5000, PC10; Abcam Ltd., Cambridge, England), and Ser10-PH3 (1: 1000, $\mathrm{H}$-0412), $\alpha$-tubulin (1:1000, DM1A), and actin (1:500, AC15) (all three from Sigma-Aldrich Chimie SNC, St. Quentin Fallavier, France), and eRF1 (1:1000, Chauvin et al ${ }^{27}$ ), followed by peroxidase-coupled secondary antibodies (1:20,000; GE Healthcare UK Ltd., Little Chalfont, Buckinghamshire, England). Membranes were revealed using SuperSignal West Pico Chemiluminescent Substrate (Pierce, Perbio Science France SA, Brebières, France).

\section{Immunofluorescence Microscopy on Primary Mouse Hepatocytes}

Hepatocytes grown on coverslips were fixed with 2\% paraformaldehyde and permeabilized with $0.5 \%$ Triton X-100 before immunolabeling using antibodies against MKIp2 (1: 1000; Echard et al $\left.{ }^{8}\right)$, Aurora B (1:250, AIM1; Cell Signaling Technology, Inc.), and GM130 (1:100; Becton Dickinson France SAS, Grenoble, France), and Alexa-conjugated secondary antibodies (1:1000; Invitrogen BP, Cergy-Pontoise, France). DNA was stained with $1 \mu \mathrm{g} / \mathrm{mL}$ Hoechst 33258, and the preparation was mounted in VectaShield medium (Vector Laboratories, Ltd., Peterborough, England). Images were obtained using an Olympus BX41 fluorescence microscope with a $\times 40$ objective (Olympus France SAS, Rungis, France) from an RTKe Spot camera (Diagnostic Instruments, Inc., Sterling Heights, MI) driven by Spot software, and were processed using Adobe Photoshop CS2 software (Adobe Systems, Inc., San Jose, CA). 

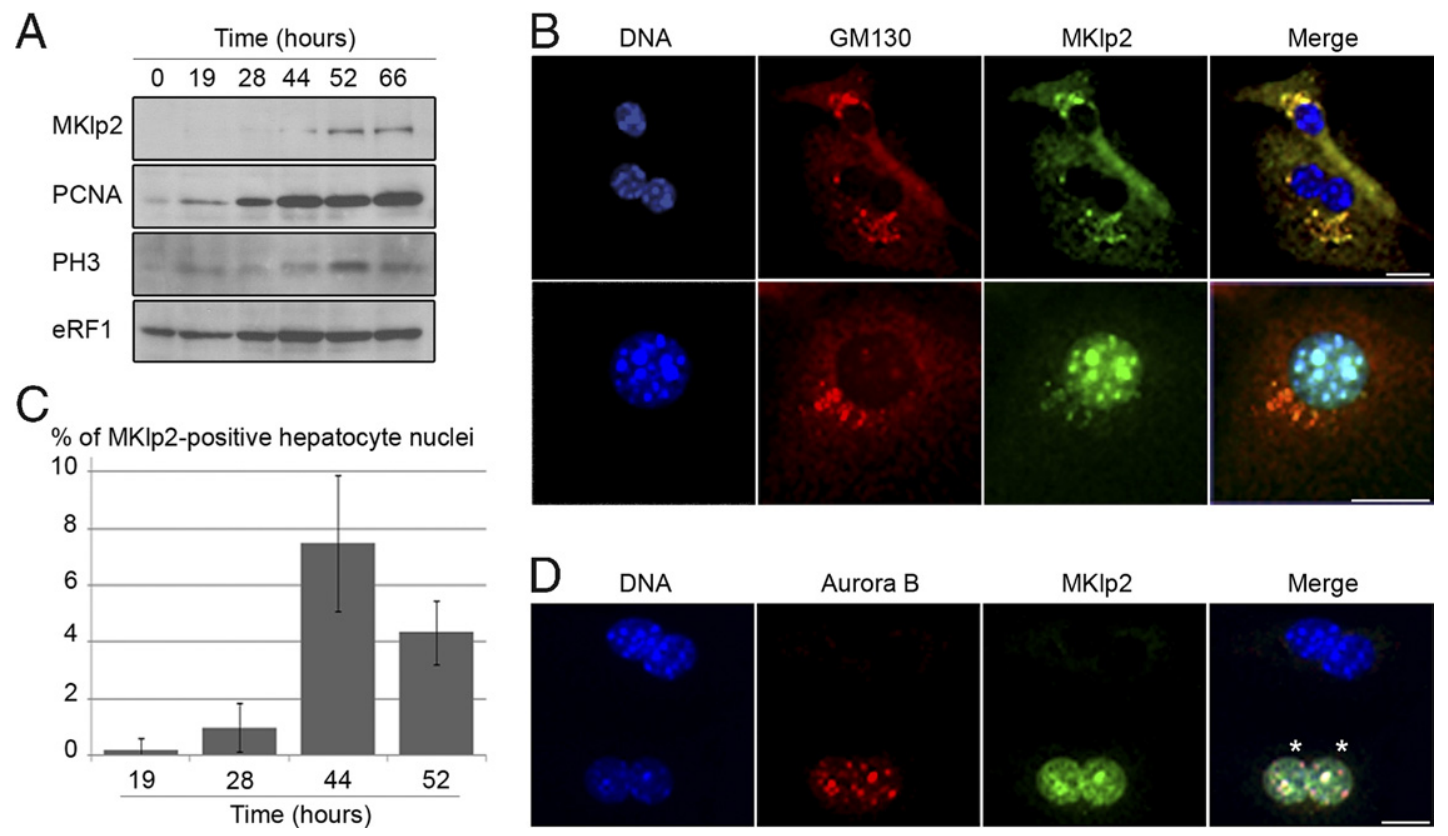

Figure 1. MKlp2 localizes to the Golgi apparatus throughout interphase and accumulates in the nucleus in the G2 phase in proliferating primary mouse hepatocytes. A: Primary mouse hepatocytes were analyzed using Western blot at the indicated times after mitogen stimulation. Liver protein extracts were analyzed in parallel using Western blot for MKlp2, PCNA, and PH3. eRF1 was used as a loading control. Results are from one of three independent experiments. B and D: Mitogen-stimulated hepatocytes were fixed and labeled using antibodies directed against the indicated proteins. DNA was counterstained with Hoechst (blue). Merge is shown on the right. Scale bar $=5 \mu \mathrm{m}$. B: MKlp2 (green) and the Golgi matrix protein GM130 (red). D: MKlp2 (green) and Aurora B kinase (red). Nuclear Aurora B labeling is typical of G2 cells and is accompanied by MKlp2 labeling (asterisks). C: Histogram shows the percentage of nuclei immunolabeled for MKlp2. All MKlp2-positive nuclei were also positive for the G2 marker Aurora B, and vice versa. Nuclei positive for only one of the two markers were not observed. Between 685 and 890 nuclei were counted at each time. Results are the mean of three independent experiments.

\section{Immunohistochemistry on Human and Mouse HCCs and Regenerating Mouse Liver}

Four-micrometer FFPE sections were cut. The sections were pretreated in a microwave oven at $750 \mathrm{~W}$ for 30 minutes in $10 \mathrm{mmol} / \mathrm{L}$ citrate buffer ( $\mathrm{pH} 6.0)$. The sections were pre-incubated with protein block (Novocastra Laboratories, Ltd) for 5 minutes, and then incubated for 30 minutes with an anti-MKIp-2 antibody (rabbit polyclonal anti-human MKIp2, Novus Biologicals, LLC, Littleton, CO, 1:100, and rabbit polyclonal anti-mouse MKIp2, ${ }^{8}$ 1:500). Post-primary block (Novolink Polymer Detection System; Novocastra Laboratories, Ltd.) was applied to the specimens for 20 minutes. Specimens were then washed with PBS ( $\mathrm{pH} 7.0$ ) for 3 minutes, and incubated with Novolink Polymer for 20 minutes. The Automate Staining System (BioGenex Laboratories, Inc., San Ramon, CA) was used. The color was developed using amino-ethyl-carbazole (AEC peroxidase substrate kit; Vector Laboratories, Ltd.). The sections were counterstained with hematoxylin.

\section{Results}

\section{MKIp2 Is Accumulated in G2 and M Phases of the Cell Cycle in Mitogen-Stimulated Primary Mouse Hepatocytes}

We first examined the timing of MKIp2 accumulation in primary mouse hepatocytes cultured in vitro in the presence of mitogens. Under these conditions, hepatocytes exit G0 and enter $\mathrm{G} 1$ on isolation, then enter the S phase at approximately 20 hours (60\% of the cells will progress through the $S$ phase within 24 hours), and demonstrate a maximal 10\% mitotic index at 50 hours after mitogen-stimulation. ${ }^{20}$ This kinetics was confirmed by monitoring the accumulation of two known cell cycle markers, proliferating cell nuclear antigen (PCNA) and phospho-histone $\mathrm{H} 3(\mathrm{PH} 3)$. PCNA is expressed from late $G 1$ to the $M$ phase, whereas $P H 3$ is specific for mitotic cells. ${ }^{28,29}$ MKlp2 was not detectable in purified nonstimulated hepatocytes (Figure 1A, time 0), as was the case for PCNA and $\mathrm{PH} 3$, in agreement with the observation that these cells are in early G1. MKIp2 began to accumulate at 44 hours after mitogen stimulation, and remained constant and high until 66 hours, whereas PCNA accumulated from 19 hours with onset of the $S$ phase, and $\mathrm{PH} 3$ reached maximal levels at 52 hours, corresponding to the peak of mitosis (Figure 1A). Thus, the timing of MKIp2 protein expression is intermediate between that of PCNA and $\mathrm{PH} 3$, which suggests that MKIp2 is primarily accumulated in the $\mathrm{G} 2$ and $M$ phases.

To confirm this hypothesis, we monitored the intracellular localization of MKIp2 during the cell cycle (Figure 1, B and C; see also Supplemental Figure S1 at http:// ajp.amjpathol.org). MKIp2 was readily detected in mitotic hepatocytes, with stage-specific re-localization of the protein from the chromosomes and the mitotic spindle to midzone microtubules, consistent with previous observations in several cell lines (see Supplemental Figure S1 at http://ajp.amjpathol.org). However, in interphase, two patterns of MKIp2 labeling were observed. The first was punctuate and perinuclear, in good agreement with the 
known association of the protein with the Golgi apparatus. ${ }^{8}$ To confirm the identity of the labeled structures, we used antibodies to GM130, a cis-Golgi matrix protein. ${ }^{30}$ Figure 1B shows the co-localization of MKIp2 and GM130 on Golgi vesicles. Such labeling was observed in both mononucleate and binucleate hepatocytes and could be detected in most interphasic hepatocytes (Figure 1B, upper panel; see also Supplemental Figure S1 at $h t t p: / /$ ajp.amjpathol.org). The second pattern of MKIp2 labeling was perinuclear and nuclear (Figure 1B, lower panel, and 1D; see also Supplemental Figure S1 at http://ajp. amjpathol.org). The percentage of MKIp2-positive hepatocyte nuclei depended, however, on the duration of mitogen stimulation, which suggests that it was dependent on the cell cycle. Less than $1 \%$ of the nuclei were labeled in hepatocytes stimulated for $<28$ hours, whereas these cells exhibited Golgi labeling (Figure 1C). Of note, MKIp2 remained undetectable by immunoblotting in these samples (Figure 1A). After 44 hours of mitogen stimulation, the percentage of cells exhibiting nuclear labeling increased to about $8 \%$, and eventually decreased at 52 hours. MKIp2 was readily detectable in these samples by immunoblotting. Therefore, detection of nuclear MKIp2 labeling coincides with the period of MKIp2 accumulation as detected by immunoblotting. To determine whether this occurs during the G2 phase of the cell cycle, we used Aurora B as a G2specific nuclear marker. Aurora $B$ is one of the major kinases that control progression through mitosis. ${ }^{2}$ In a wide variety of cell types, nuclear accumulation of Aurora B occurs in $\mathrm{G} 2$ before mitosis. Aurora B then persists throughout mitosis, and is degraded on mitotic exit. Immunofluorescence data (Figure 1D) demonstrated that Aurora B and MKIp2 are co-expressed in the nucleus of mitogen-stimulated hepatocytes. We observed that all MKIp2-positive nuclei were also positive for Aurora B, and vice versa, throughout the culture period, with a maximum 44 hours after mitogen stimulation (Figure 1C). These results demonstrate that MKIp2 is accumulated during G2 in the nucleus of mitogen-stimulated hepatocytes.

\section{MKIp2 Is Strongly Accumulated During Mouse Liver Regeneration}

Liver regeneration after partial hepatectomy has been widely used to study the hepatocyte cell cycle because it provides a physiologic setting of normal cell division. After partial hepatectomy in the mouse, hepatocytes undergo a first round of synchronized cell division, with peak DNA synthesis occurring at 36 hours after partial hepatectomy and a maximal mitotic index at 48 hours after partial hepatectomy. ${ }^{31}$ A second wave of hepatocyte cell division occurs during the next 48 hours but is less synchronous and overlaps with division of other liver cell types. To determine the expression pattern of MKIp2 during liver regeneration, livers were collected between 32 and 96 hours after partial hepatectomy, representing the interval of two hepatocyte cell division cycles. To monitor the hepatocyte cell cycle, liver sections were analyzed for Ki-67 immunolabeling of hepatocyte nuclei (Figure 2A). Ki-67 is absent in quiescent cells but is
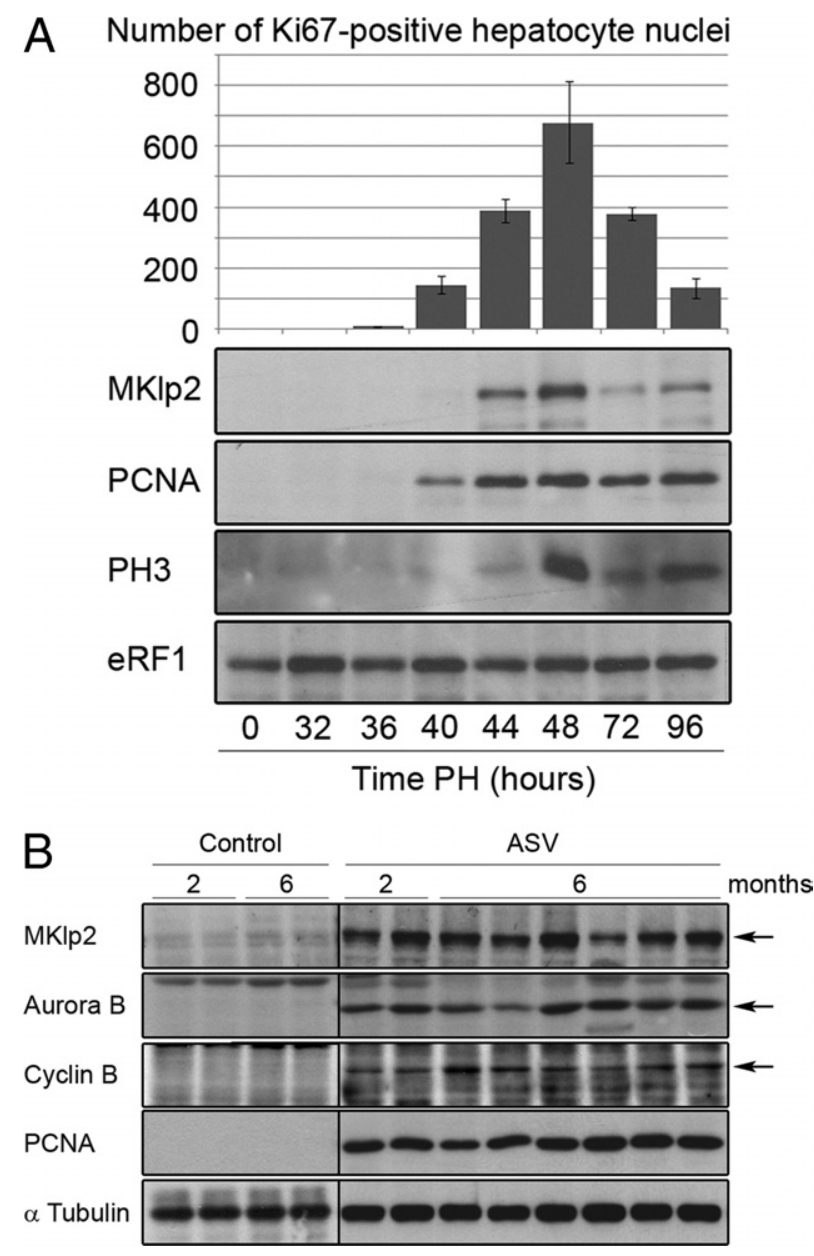

Figure 2. MKlp2 strongly accumulates during mouse liver regeneration and hepatocarcinogenesis. A: Upper panel, Histogram shows the time course of hepatocyte proliferation during liver regeneration assessed by Ki-67 immunolabeling. Positive Ki-67 hepatocyte nuclei were counted in 10 consecutive fields from each liver section at the indicated time after partial hepatectomy and are expressed as mean \pm SEM. Lower panel, Liver protein extracts were analyzed in parallel using Western blot for MKlp2, PCNA, and PH3. eRF1 was used as a loading control. B: Mouse liver protein extracts were analyzed in parallel using Western blot for MKlp2, Aurora B, cyclin B, and PCNA. $\alpha-$ Tubulin was used as a loading control. Control, 2- and 6-month-old nontransgenic males; ASV, male transgenic mice with nuclear atypias in the liver ( 2 months old) and HCC (6 months old).

accumulated in late $\mathrm{G} 1$ and is present in all subsequent cell cycle phases. In the present study, we observed that the number of Ki-67-positive hepatocyte nuclei increased from 36 to 48 hours after partial hepatectomy, then gradually decreased as hepatocytes progressively ceased to proliferate, and no Ki-67-positive nuclei were detected in quiescent liver (time 0). Therefore, hepatocytes reached late $\mathrm{G} 1$ at 36 hours after partial hepatectomy and afterward. As expected, neither PCNA nor PH3 was detectable in quiescent liver or in regenerating liver until 36 hours after partial hepatectomy. Levels of PCNA increased from 40 hours, to reach a maximum at 48 hours after partial hepatectomy, and remained elevated. This indicated that the hepatocyte $S$ phase started at approximately 40 hours after partial hepatectomy. Maximal levels of $\mathrm{PH} 3$ were observed primarily at 48 and 96 hours after partial hepatectomy, representing the two successive waves of hepatocyte mitosis. The timing of MKIp2 
A

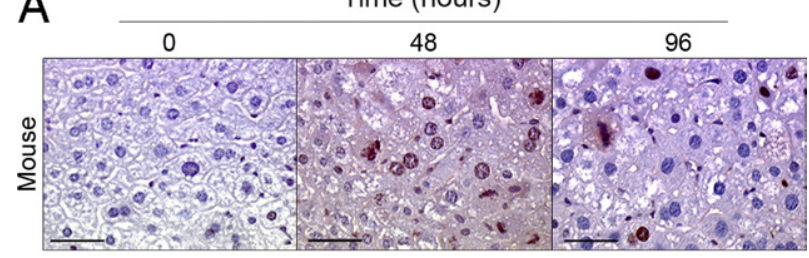

B



Figure 3. Mklp2 immunolabeling in regenerating mouse liver and in murine and human HCC. Tissue sections from regenerating liver and HCC were labeled using anti-Mklp2 antibodies, and were counterstained with hematoxylin. Representative images are shown. A: Regenerating mouse liver at the indicated time after hepatectomy. Typical nuclear hepatocyte labeling is observed at 48 hours after hepatectomy. Hepatocytes in mitosis exhibit strongly labeled chromosomes (best observed at 96 hours after hepatectomy). No labeling is detected in quiescent liver (time 0). Scale bar $=50 \mu \mathrm{m}$. B: Mouse and human liver tumors. Typical nuclear hepatocyte labeling is observed in liver tumors, representing approximately $10 \%$ and $40 \%$ of the nuclei in mouse and human tumors, respectively, whereas this labeling was absent in control liver. Scale bar $=100 \mu \mathrm{m}$.

protein expression was intermediate between that of PCNA and PH3 (Figure 2A). MKIp2 expression was undetectable in quiescent liver (time 0) but dramatically increased at 44 hours, after the increase in PCNA, and reached a maximum at 48 hours after partial hepatectomy, coincident with maximal accumulation of $\mathrm{PH} 3$. MKIp2 protein levels then decreased at 72 hours, and increased again at 96 hours after partial hepatectomy, coincident with the second wave of hepatocyte mitosis. Accumulation of MKIp2 in the hepatocyte population of cells in the regenerating liver was confirmed by in situ analysis of fixed liver samples. At 48 hours after partial hepatectomy, approximately $40 \%$ of the nuclei were strongly positive for MKIp2 labeling, whereas no labeling was observed in quiescent liver (Figure 3A). Mitotic hepatocytes with labeled chromosomes were also observed at 48 and 96 hours after partial hepatectomy. These observations are consistent with our finding that MKIp2 mostly accumulates in the G2 phase, and mitosis in mitogenstimulated hepatocytes proliferating in vitro.

\section{MKIp2 Is Overexpressed During Mouse Liver Transformation}

ASV transgenic mice provide a well-characterized model of large $T$ antigen-induced hepatocarcinogenesis. Male transgenic mice develop hepatocellular atypias and abnormal levels of mitosis in the liver at age 2 months, followed by the emergence of diffuse hepatocellular carcinoma in all animals at age 6 months. The presence of proliferating cells in these tissues is characterized by overexpression of the cell cycle proteins cyclin B and PCNA (Figure 2B). We observed that MKIp2 is overexpressed in both preneoplastic livers at age 2 months and hepatocarcinomas in 6-month-old male transgenic mice (Figure 2B), whereas it remains undetectable in control liver from nontransgenic males (Figure 2B) and female littermates (not shown). Similarly, the mitotic kinase Aurora B was also increased. Accumulation of MKIp2 in transformed liver was finally confirmed by in situ analysis of fixed liver samples. Approximately $10 \%$ of the nuclei were strongly positive for MKlp2 labeling in hepatocarcinoma sections from transgenic mice, whereas no labeling was observed in nontransgenic normal liver (Figure 3B).

\section{MKIp2 Is Overexpressed in Human Hepatoma Cell Lines}

We first analyzed MKIp2 expression in a panel of human hepatoma cell lines and in normal human hepatocytes in primary culture (Figure 4A). It was observed that MKIp2 is undetectable in normal primary hepatocytes but is expressed at intermediate levels in PLC/PRF/5 and HepG2 to high levels in $\mathrm{HuH} 6, \mathrm{HuH} 7$ and Hep3B cell lines. Microscopic analysis demonstrated that the intracellular localization of MKIp2 followed the pattern observed in primary mouse hepatocytes (exemplified for the HuH6 cell line in Supplemental Figure S2, available at http://ajp. amjpathol.org). We then used RNA interference to investigate the function of MKIp2 in $\mathrm{HuH} 6$ and $\mathrm{HuH} 7$ cells. The $\mathrm{HuH} 7$ cell line is mutated in the TP53 gene, and cells tend to become spontaneously polyploid. Knockdown of MKIp2 was efficient in both cell lines (Figure 4B). This was accompanied in $\mathrm{HuH} 6$ cells by the appearance of polyploid cells, with a slight increase in the percentage of cells with $>4 n$ DNA content, from $2.2 \%$ to $3.8 \%$. A dramatic effect was obtained in $\mathrm{HuH} 7$ cells, passing from $26.3 \%$ to $73.2 \%$ of cells with $>4$ n DNA content on MKIp2 knockdown. In these two cell lines, modeling of the cell cycle predicted the presence of cycling tetraploid cells at higher frequency after MKIp2 knockdown. Microscopic analysis indicated that cells down-regulated for MKIp2 exhibited abnormal mitoses, with mispositioned chromosomes in metaphase and reduced furrow ingression in telophase and cytokinesis (see Supplemental Figure S2 at at http://ajp.amjpathol.org). Therefore, MKIp2 is required to prevent polyploidization and to promote cytokinesis in human hepatoma cells. However, no obvious increase in the sub-G1 population and no apoptosis were detected after MKIp2 knockdown (see Supplemental Figure S3A at http://ajp.amjpathol.org).

To investigate long-term effects of MKIp2 knockdown in hepatoma cells, we selected for stable expression of MKIp2 shRNA in HuH6 cells. The number of macro colonies ( $>50$ cells) formed after 14 days of selection was severely reduced (Figure $4 \mathrm{C}$ ). In addition, we observed 
A

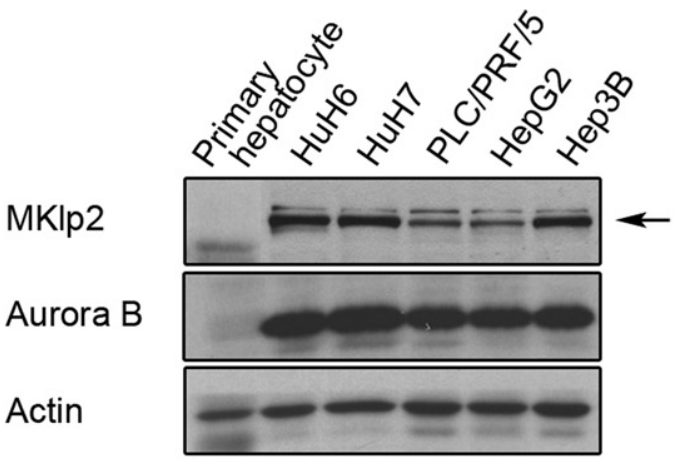

B

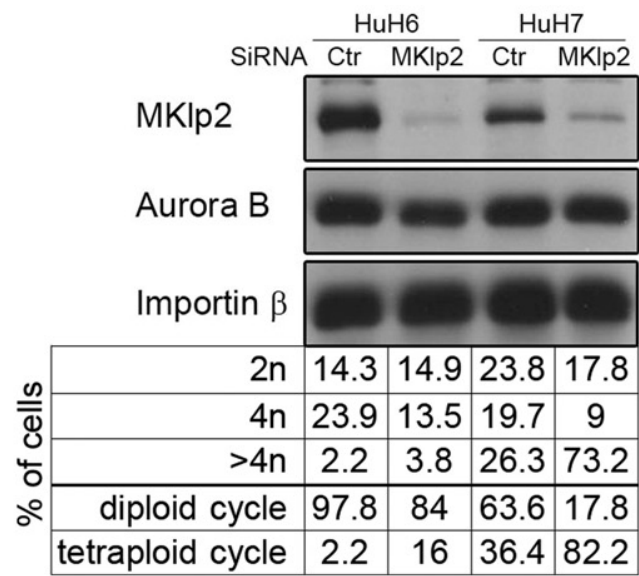

C

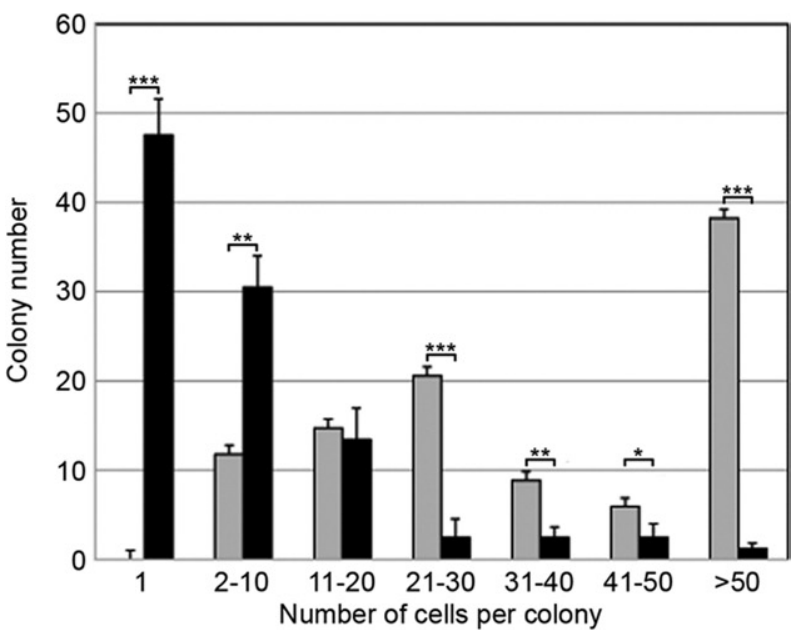

Figure 4. Hepatoma cells with reduced MKlp2 levels demonstrate polyploidization. A and B: Western blot analysis of normal human primary hepatocytes and the indicated hepatoma cell lines. A: Detection of MKlp2 and Aurora B. Actin was used as a loading control. B: HuH6 and HuH7 hepatoma cells transfected for 36 hours with pSuper plasmids expressing control (Ctr) or MKlp2 sh-RNAs (Mklp2) were analyzed for MKlp2, Aurora B, and importin- $\beta$ (loading control). Cells were analyzed in parallel using flow cytometry. Values represent percentage of cells with the indicated DNA contents, either as separate classes (2n, $4 \mathrm{n}$, and $>4 \mathrm{n}$ DNA contents) or as two overlapping cell cycles (diploid and tetraploid). C: Colony formation assay in HuH6 hepatoma cells stably expressing MKlp2 (black) and control (gray) shRNA and neo expressing vector. Quantification of the number of colonies per $25-\mathrm{cm}^{2}$ flask demonstrates a significant reduction in the number of macrocolonies ( $n>50$ cells per colony) and persistence of single cells and microcolonies ( $n<20$ cells per colony) on down-regulation of MKlp2. The experiment was performed in triplicate, and the two-tailed Student's $t$-test was used to evaluate statistical significance $\left({ }^{*} P<0.05 ;{ }^{* *} P<0.005 ;{ }^{* * * *} P<0.0005\right)$.

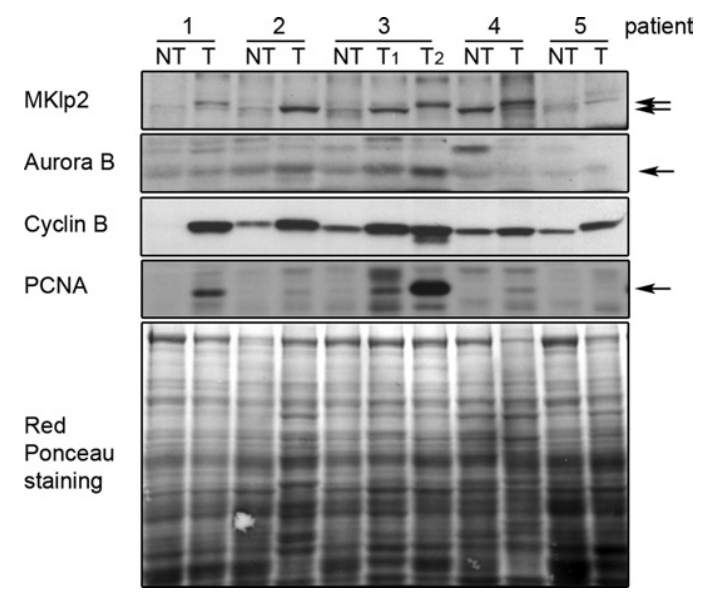

Figure 5. MKlp2 strongly accumulates during human hepatocarcinogenesis Tumoral (T) and nontumoral (NT) adjacent liver tissue from five patients with HCC were analyzed using Western blot for MKlp2, Aurora B, cyclin B, and PCNA. Equivalent protein loading was assessed with red Ponceau staining. Patients 1,3 , and 4 were without fibrosis. Patients 2 and 5 had hepatitis $C$ virus infection and cirrhosis. Two separate tumors (T1 and T2) were obtained from the same patient.

the persistence of binucleate cells and cells with severe nuclear abnormalities (enlarged multilobulated nuclei and micronuclei) consistent with a cytokinesis defect. They were observed as singlets and in microcolonies of $<20$ cells (see Supplemental Figure S3, B-E, at http:// ajp.amjpathol.org). This result indicated that down-regulation of MKIp2 is incompatible with sustained cell proliferation.

\section{MKIp2 Is Overexpressed in Specimens of Human HCC}

To determine whether MKIp2 overexpression is a general feature of liver oncogenic transformation, we evaluated the levels of MKIp2 in a small series of human HCC specimens in comparison with nontumoral adjacent liver tissue. MKIp2 was largely overexpressed in four tumor samples (from patients 1, 2, and 3) compared with the adjacent tissue, independent of liver status (healthy or cirrhotic liver) (Figure 5). In one patient (patient 4), MKIp2 was similarly expressed in both tumor and nontumor samples. MKIp2 was barely detectable in the last specimen analyzed (patient 5). There was good but not absolute correlation between MKIp2 overexpression and the presence of proliferating cells in the samples, as judged from accumulation of the cell cycle proteins cyclin $B$ and PCNA. MKIp2 mobility at SDS-PAGE was altered in four tumor samples (samples 1, 3T2, 4, and 5). In patient 3, this alteration was observed in one tumor (T2) but not the other (T1). These observations suggest that MKIp2 could be the subject of post-translational modifications specific to a subset of HCCs.

Overexpression of the mitotic kinase Aurora B in tumoral samples compared with nontumoral samples was observed in only two patients (patients 2 and 3). Expression without accumulation in the tumor was observed in one patient (patient 1). Aurora B was barely detectable in samples from two patients (patients 4 and 5). Accumu- 
lation of MKIp2 in human HCCs was confirmed using immunohistochemistry. A strong nuclear labeling for MKIp2 in human HCC tissue sections was observed in approximately $40 \%$ of the cells, and the adjacent nontumoral tissue was unlabeled (Figure 3B). Altogether, these observations demonstrate that accumulation of nuclear MKIp2 is a prominent feature of tumoral liver cells.

\section{MKIp2 Encoding KIF20A mRNA Is Increased in a Wide Range of Human HCCs, with the Highest Levels Linked to Chromosome Instability}

To determine whether overexpression of MKIp2 is definitely linked to human hepatocarcinogenesis, we used quantitative RT-PCR to measure the level of KIF20A mRNA in a series of 125 tumors that have been already classified into six subgroups associated with clinical and genetic characteristics. ${ }^{24} \mathrm{KIF} 20 \mathrm{~A}$ mRNA was increased 8- to 40-fold in all HCC subgroups compared with normal liver tissues (Figure 6A). The highest levels of expression were observed in subgroups G1, G2, and G3, which are all significantly associated with chromosome instability and increased cell proliferation.

Subgroup G3 demonstrated the highest value, with a 40-fold increase in KIF20A mRNA. This subgroup is defined by up-regulation of several checkpoint proteins involved in cell-cycle control, TP53 mutation, and absence of HBV infection. We then analyzed the association between KIF20A mRNA accumulation and clinical factors and genetic alterations. KIF20A mRNA levels were significantly higher in HCCs harboring mutations in TP53 (subgroups G2 and G3), infected with hepatitis B virus (subgroups G1 and G2), or associated with high $\alpha$-fetoprotein levels (Figure 6B). Significantly higher KIF20A mRNA levels were also observed in poorly differentiated and undifferentiated tumors (Edmondson grades III and IV, respectively) compared with well-differentiated and moderately differentiated tumors (Edmondson grades I and II, respectively). However, KIF20A mRNA levels were not significantly linked to alcohol, cirrhosis, hepatitis $\mathrm{C}$ virus infection, or $\beta$-catenin mutations (not shown). Therefore, high levels of KIF20A mRNA were significantly linked to the most proliferative and undifferentiated HCCs.

\section{Discussion}

HCC is a common fatal malignant tumor that is characterized by a high incidence of recurrence, development of resistance to chemotherapy, and low sensitivity to radiation therapy. Identification of novel biomarkers for predictive medicine is required to better classify HCCs according to the probability of recurrence after chemical therapy or surgical resection. In the present study, we demonstrate that the mitotic kinesin MKIp2 is deregulated during human and mouse hepatocarcinogenesis. MKIp2 encoding KIF20A mRNA is significantly overexpressed in human HCCs, with the highest levels linked to chromosome instability. At the protein level, MKIp2 accumulation
A Relative KIF20A mRNA expression

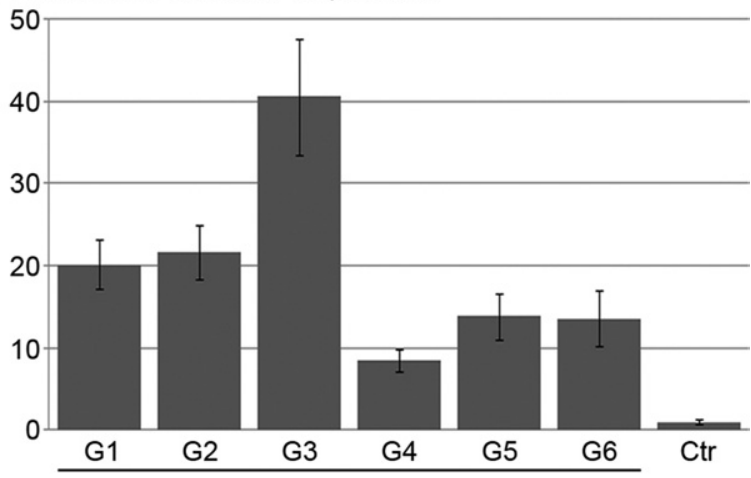

G1-G6 transcriptomic subgroups

B Relative KIF20A mRNA expression

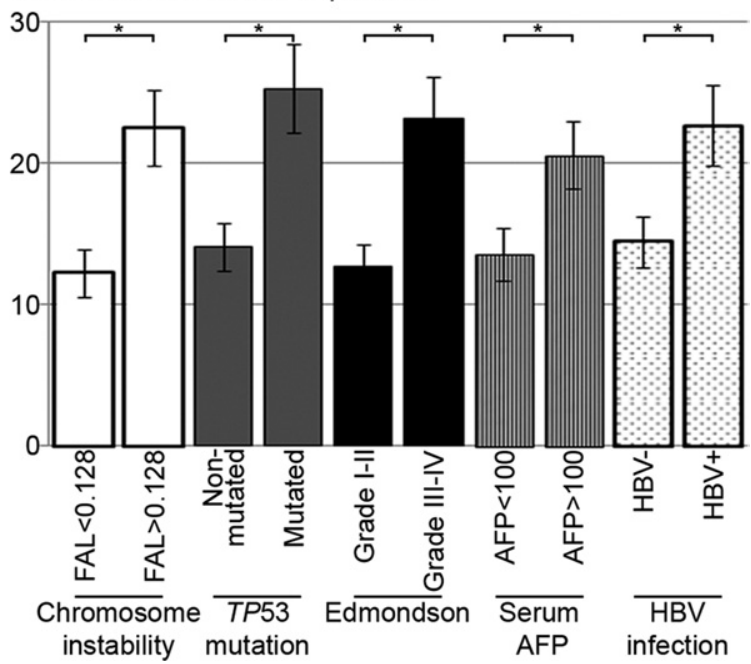

Figure 6. MKlp2 encoding KIF20A mRNA is dramatically increased in human HCCs and is linked to tumor aggressiveness. KIF2OA gene expression was quantified using RT-PCR. Results were first normalized to control ribosomal $18 \mathrm{~S}$ in all samples. A: Results in HCC samples are expressed as a ratio relative to the mean expression level in six control liver samples (Ctr). Bars show the values as mean \pm SEM. Significance determined using the nonparametric Mann-Whitney $U$ test was $P=0.0001$ (difference was considered significant at $P<0.05$ ). B: Monoparametric analysis of KIF20A mRNA expression in HCCs with chromosome instability [FAL, frequency of allelic loss (white, $P=0.0001$ ), TP53 gene mutation (gray, $P=0.0001$ ), Edmondson histologic grade (black, $P=0.0009$ ), serum $\alpha$-fetoprotein concentration (AFP, hatched, $P=0.001$ ), and hepatitis B virus infection (dotted, $P=0.001$ ). Bars show the values as mean \pm SEM. ${ }^{*} P<0.05$.

was also observed in human hepatoma cell lines and in human HCC specimens irrespective of whether the underlying liver was cirrhotic or was affected with periportal fibrosis (data not shown). We observed increased MKIp2 protein expression in HCCs from a murine model of hepatocarcinogenesis, and in this model, MKIp2 overexpression was detected at preneoplastic stages. In addition, we demonstrated that MKIp2 is cell cycle-regulated in normal hepatocytes, being accumulated in the G2 phase and in mitosis in primary hepatocytes. In contrast, MKIp2 is barely detectable in quiescent liver and hepatocytes. RNA interference assays demonstrated that MKIp2 was functional in human hepatoma cells and was required to prevent polyploidization. MKIp2 knockdown did not induce apoptosis, but inhibited cell proliferation on stable expression. 
Our observations in mouse primary hepatocytes demonstrate that MKIp2 nuclear accumulation occurs in G2 coincident with the mitotic kinase Aurora B. The gene encoding Aurora B was observed to be significantly overexpressed in a recent genome-wide microarray analysis of 107 human HCCs, and was the most significant predictor of aggressive recurrence. ${ }^{32}$ In that study, genes encoding several cytokinesis- and mitotic spindle-associated proteins including KIF20A were observed to be up-regulated in HCCs with an aggressive pattern of recurrence. Our observation that MKIp2 is overexpressed at the mRNA level in human HCCs is in good agreement with that study and indicates that MKIp2 overexpression resulted from either enhanced gene transcription or mRNA stabilization during liver transformation.

Several lines of evidence indicate that the two genes encoding MKIp2 and Aurora B are co-regulated, although they are located on different chromosomes. The promoter regions of both genes share common regulatory sequences such as the cell cycle-dependent element and cell cycle homology region, which are able to bind specific E2F/DP complexes mediating both transcriptional repression in $\mathrm{G} 0$ through $\mathrm{pRb}$ and activation in $\mathrm{S} / \mathrm{G2}$. $^{6,33}$ Liver-specific inactivation of $\mathrm{pRb}$ in the mouse, which mimics the situation observed at high frequency in human HCCs, results in deregulation of E2F target genes and aberrant ploidy. ${ }^{16,34}$ In the present study, we demonstrate that alteration of the pRb and p53 tumor-suppressor protein mediated by large $T$ antigen in the mouse liver is sufficient to up-regulate both MKIp2 and Aurora B. This up-regulation is observed in preneoplastic livers and persists in HCCs, which suggests a role for MKIp2 at both early and later stages of liver carcinogenesis. In good agreement with these observations in the mouse, in humans, high levels of MKIp2 encoding mRNA are observed in HCCs with mutated TP53. Studies of chromatin immunoprecipitation have demonstrated that the forkhead transcription factor FoxM1 is an essential transcriptional regulator of both MKIp2 and Aurora B. ${ }^{35}$ Elevated expression of FoxM1 has been demonstrated in human HCCs, and, conversely, FoxM1 $1^{-1-}$ mice exhibit defects in tissues that normally become polyploid such as liver and heart, with an up to 50-fold increase in DNA content. ${ }^{36}$ Inhibition of FoxM1 by a cell penetrating ARF peptide was recently demonstrated to induce apoptosis in human HCC cell lines and mouse models. ${ }^{37}$ Therefore, deregulation of the transcriptional circuitry depending on both $\mathrm{pRb} / \mathrm{E} 2 \mathrm{~F}$ and Fox M1 leads to up-regulation of at least the two target genes Aurora $B$ and KIF20A, which may be sufficient to impair mitosis and cytokinesis, leading to aberrant ploidy and genomic instability.

Transcriptome classification of human HCCs demonstrated that groups associated with a high rate of chromosomal instability were enriched with cell cycle, proliferation, and DNA metabolism genes. ${ }^{24}$ In the same series, we observed the highest levels of KIF20A mRNA in subgroup G3, which includes tumors specified by mutation of TP53, a global overexpression of cell cycle genes, and inactivation of the $\mathrm{pRb}$ pathway through CDKN2A promoter methylation. This result is in agree- ment with KIF20A belonging to the cell cycle gene family and being a target of E2F transcription factor.

In conclusion, our observation that MKIp2 accumulates in normal proliferating hepatocytes and liver cancer cells suggests that MKIp2 contributes to both normal and malignant hepatocyte proliferation. Furthermore, our data indicate that MKIp2 should be considered a new predictor candidate for HCC. Specific and stable inhibition of MKIp2 (now feasible using the new selective inhibitor paprotrain ${ }^{38}$ ) seems to be a new antiproliferative strategy in the treatment of liver cancer.

\section{Acknowledgments}

We thank Elisabeth Moreau for technical assistance, Thomas Lelli, Thomas Boudier, Annie Munier, and Virginie Georget (the Imaging and Flow Cytometry core facility at IFR 83) and Anne-Marie Faussat (IFR 115) for their contributions to image acquisition and flow cytometry analysis, Isabelle Guillet-Deniau for helpful advice about liver protein extraction, Bruno Goud for providing antiserum against MKIp2, Olivier Jean-Jean for antibodies specific to eRF1, Colette Rey for assistance with partial hepatectomy, and Christele Desbois-Mouthon for providing the hepatoma cell lines.

\section{References}

1. Zhu C, Zhao J, Bibikova M, Leverson JD, Bossy-Wetzel E, Fan JB, Abraham RT, Jiang W: Functional analysis of human microtubulebased motor proteins, the kinesins and dyneins, in mitosis/cytokinesis using RNA interference. Mol Biol Cell 2005, 16:3187-3199

2. Barr FA, Gruneberg U: Cytokinesis: placing and making the final cut. Cell 2007, 131:847-860

3. Neef R, Preisinger C, Sutcliffe J, Kopajtich R, Nigg EA, Mayer TU, Barr FA: Phosphorylation of mitotic kinesin-like protein 2 by polo-like kinase 1 is required for cytokinesis. J Cell Biol 2003, 162:863-875

4. Gruneberg U, Neef R, Honda R, Nigg EA, Barr FA: Relocation of Aurora $B$ from centromeres to the central spindle at the metaphase to anaphase transition requires MKIp2. J Cell Biol 2004, 166:167-172

5. Neef R, Klein UR, Kopajtich R, Barr FA: Cooperation between mitotic kinesins controls the late stages of cytokinesis. Curr Biol 2006, 16: 301-307

6. Fontijn RD, Goud B, Echard A, Jollivet F, van Marle J, Pannekoek H, Horrevoets AJ: The human kinesin-like protein RB6K is under tight cell cycle control and is essential for cytokinesis. Mol Cell Biol 2001, 21:2944-2955

7. Hill E, Clarke M, Barr FA: The Rab6-binding kinesin, Rab6-KIFL, is required for cytokinesis. EMBO J 2000, 19:5711-5719

8. Echard A, Jollivet F, Martinez O, Lacapere JJ, Rousselet A, JanoueixLerosey I, Goud B: Interaction of a Golgi-associated kinesin-like protein with Rab6. Science 1998, 279:580-585

9. Lai F, Fernald AA, Zhao N, Le Beau MM: cDNA cloning, expression pattern, genomic structure and chromosomal location of RAB6-KIFL, a human kinesin-like gene. Gene 2000, 248:117-125

10. Fausto N, Campbell JS, Riehle KJ: Liver regeneration. Hepatology 2006, 43:S45-S53

11. Markey MP, Angus SP, Strobeck MW, Williams SL, Gunawardena RW, Aronow BJ, Knudsen ES: Unbiased analysis of RB-mediated transcriptional repression identifies novel targets and distinctions from E2F action. Cancer Res 2002, 62:6587-6597

12. Lazarevich NL, Cheremnova OA, Varga EV, Ovchinnikov DA, Kudrjavtseva EI, Morozova OV, Fleishman DI, Engelhardt NV, Duncan SA: Progression of HCC in mice is associated with a downregulation in the expression of hepatocyte nuclear factors. Hepatology 2004 , 39:1038-1047 
13. Rosty C, Sheffer M, Tsafrir D, Stransky N, Tsafrir I, Peter M, de Cremoux P, de La Rochefordiere A, Salmon R, Dorval T, Thiery JP, Couturier J, Radvanyi F, Domany E, Sastre-Garau X: Identification of a proliferation gene cluster associated with HPV E6/E7 expression level and viral DNA load in invasive cervical carcinoma. Oncogene 2005, 24:7094-7104

14. Karstensen B, Poppelreuther S, Bonin M, Walter M, Iftner T, Stubenrauch $\mathrm{F}$ : Gene expression profiles reveal an upregulation of E2F and downregulation of interferon targets by HPV18 but no changes between keratinocytes with integrated or episomal viral genomes. Virology 2006, 353:200-209

15. Eguchi $T$, Takaki $T$, Itadani $H$, Kotani $H$ : RB silencing compromises the DNA damage-induced G2/M checkpoint and causes deregulated expression of the ECT2 oncogene. Oncogene 2007, 26:509-520

16. Mayhew CN, Carter SL, Fox SR, Sexton CR, Reed CA, Srinivasan SV, Liu X, Wikenheiser-Brokamp K, Boivin GP, Lee JS, Aronow BJ, Thorgeirsson SS, Knudsen ES: RB loss abrogates cell cycle control and genome integrity to promote liver tumorigenesis. Gastroenterology 2007, 133:976-984

17. Odom DT, Zizlsperger N, Gordon DB, Bell GW, Rinaldi NJ, Murray HL, Volkert TL, Schreiber J, Rolfe PA, Gifford DK, Fraenkel E, Bell GI, Young RA: Control of pancreas and liver gene expression by HNF transcription factors. Science 2004, 303:1378-1381

18. Dubois N, Bennoun M, Allemand I, Molina T, Grimber G, DaudetMonsac M, Abelanet R, Briand P: Time-course development of differentiated hepatocarcinoma and lung metastasis in transgenic mice. J Hepatol 1991, 13:227-239

19. Boissan M, Beurel E, Wendum D, Rey C, Lecluse Y, Housset C, Lacombe ML, Desbois-Mouthon C: Overexpression of insulin receptor substrate-2 in human and murine hepatocellular carcinoma. Am J Pathol 2005, 167:869-877

20. Guidotti JE, Bregerie O, Robert A, Debey P, Brechot C, Desdouets C: Liver cell polyploidization: a pivotal role for binuclear hepatocytes. J Biol Chem 2003, 278:19095-19101

21. Lakehal F, Dansette PM, Becquemont L, Lasnier E, Delelo R, Balladur $\mathrm{P}$, Poupon R, Beaune PH, Housset C: Indirect cytotoxicity of flucloxacillin toward human biliary epithelium via metabolite formation in hepatocytes. Chem Res Toxicol 2001, 14:694-701

22. Brummelkamp TR, Bernards R, Agami R: A system for stable expression of short interfering RNAs in mammalian cells. Science 2002 , 296:550-553

23. Bellanger S, de Gramont A, Sobczak-Thepot J: Cyclin B2 suppresses mitotic failure and DNA re-replication in human somatic cells knocked down for both cyclins B1 and B2. Oncogene 2007, 26:7175-7184

24. Boyault S, Rickman DS, de Reynies A, Balabaud C, Rebouissou S, Jeannot E, Herault A, Saric J, Belghiti J, Franco D, Bioulac-Sage P, Laurent-Puig P, Zucman-Rossi J: Transcriptome classification of HCC is related to gene alterations and to new therapeutic targets. Hepatology 2007, 45:42-52

25. Bioulac-Sage P, Rebouissou S, Sa Cunha A, Jeannot E, Lepreux S, Blanc JF, Blanche H, Le Bail B, Saric J, Laurent-Puig P, Balabaud C,
Zucman-Rossi J: Clinical, morphologic, and molecular features defining so-called telangiectatic focal nodular hyperplasias of the liver. Gastroenterology 2005, 128:1211-1218

26. Livak KJ, Schmittgen TD: Analysis of relative gene expression data using real-time quantitative PCR and the 2(-Delta Delta C(T)) Method. Methods 2001, 25:402-408

27. Chauvin C, Salhi S, Le Goff C, Viranaicken W, Diop D, Jean-Jean O: Involvement of human release factors eRF3a and eRF3b in translation termination and regulation of the termination complex formation. $\mathrm{Mol}$ Cell Biol 2005, 25:5801-5811

28. Connolly KM, Bogdanffy MS: Evaluation of proliferating cell nuclear antigen (PCNA) as an endogenous marker of cell proliferation in rat liver: a dual-stain comparison with 5-bromo-2'-deoxyuridine. J Histochem Cytochem 1993, 41:1-6

29. Juan G, Traganos F, James WM, Ray JM, Roberge M, Sauve DM, Anderson H, Darzynkiewicz Z: Histone H3 phosphorylation and expression of cyclins A and B1 measured in individual cells during their progression through G2 and mitosis. Cytometry 1998, 32:71-77

30. Nakamura N, Rabouille C, Watson R, Nilsson T, Hui N, Slusarewicz P, Kreis TE, Warren G: Characterization of a cis-Golgi matrix protein. GM130. J Cell Biol 1995, 131:1715-1726

31. Matsuo T, Yamaguchi S, Mitsui S, Emi A, Shimoda F, Okamura H: Control mechanism of the circadian clock for timing of cell division in vivo. Science 2003, 302:255-259

32. Tanaka S, Arii S, Yasen M, Mogushi K, Su NT, Zhao C, Imoto I, Eishi $\mathrm{Y}$, Inazawa J, Miki $\mathrm{Y}$, Tanaka $\mathrm{H}$ : Aurora kinase $\mathrm{B}$ is a predictive factor for the aggressive recurrence of hepatocellular carcinoma after curative hepatectomy. Br J Surg 2008, 95:611-619

33. Kimura M, Uchida C, Takano Y, Kitagawa M, Okano Y: Cell cycledependent regulation of the human Aurora B promoter. Biochem Biophys Res Commun 2004, 316:930-936

34. Mayhew CN, Bosco EE, Fox SR, Okaya T, Tarapore P, Schwemberger SJ, Babcock GF, Lentsch AB, Fukasawa K, Knudsen ES: Liverspecific pRB loss results in ectopic cell cycle entry and aberrant ploidy. Cancer Res 2005, 65:4568-4577

35. Wonsey DR, Follettie MT: Loss of the forkhead transcription factor FoxM1 causes centrosome amplification and mitotic catastrophe. Cancer Res 2005, 65:5181-5189

36. Korver W, Schilham MW, Moerer P, van den Hoff MJ, Dam K, Lamers $\mathrm{WH}$, Medema $\mathrm{RH}$, Clevers $\mathrm{H}$ : Uncoupling of $\mathrm{S}$ phase and mitosis in cardiomyocytes and hepatocytes lacking the winged-helix transcription factor Trident. Curr Biol 1998, 8:1327-1330

37. Gusarova GA, Wang IC, Major ML, Kalinichenko VV, Ackerson T, Petrovic V, Costa $\mathrm{RH}$ : A cell-penetrating ARF peptide inhibitor of FoxM1 in mouse hepatocellular carcinoma treatment. J Clin Invest 2007, 117:99-111

38. Tcherniuk S, Skoufias DA, Labriere C, Rath O, Gueritte F, Guillou C, Kozielski F: Relocation of Aurora B and survivin from centromeres to the central spindle impaired by a kinesin-specific MKLP-2 inhibitor. Angew Chem Int Ed Engl 2010, 49:8228-8231 